\title{
Challenges during the transition from child and adolescent mental health services to adult mental health services
}

\author{
Gaelle Hendrickx, ${ }_{1}^{1}$ (D) Veronique De Roeck, ${ }^{2}$ Athanasios Maras, ${ }^{3}$ Gwen Dieleman, ${ }^{4}$ \\ Suzanne Gerritsen, ${ }^{4}$ Diane Purper-Ouakil, ${ }^{5}$ Frédérick Russet, ${ }^{5}$ Renate Schepker, ${ }^{6}$ \\ Giulia Signorini, ${ }^{7}$ Swaran Preet Singh, ${ }^{8}$ Cathy Street, ${ }^{8}$ Helena Tuomainen, ${ }^{8}$ \\ Sabine Tremmery ${ }^{2}$ on behalf of MILESTONE consortium
}

BJPsych Bulletin (2020) 44, 163-168, doi:10.1192/bjb.2019.85

\author{
${ }^{1}$ Department of Neurosciences, Centre \\ for Clinical Psychiatry, KU Leuven, \\ Belgium; ${ }^{2}$ Department of \\ Neurosciences, Child and Adolescent \\ Psychiatry, KU Leuven, Belgium; ${ }^{3}$ Yulius \\ Academy, Yulius Mental Health \\ Organization, The Netherlands; \\ ${ }^{4}$ Department of Child and Adolescent \\ Psychiatry and Psychology, Erasmus \\ Medical Center, The Netherlands; ${ }^{5}$ Child \\ and Adolescent Psychiatry Unit, \\ University Hospital of Montpellier, \\ France; ${ }^{6}$ Centre for Psychiatry South- \\ Wuerttemberg, Germany; ${ }^{7}$ Psychiatric \\ Epidemiology and Evaluation Unit, Saint \\ John of God Clinical Research Center, \\ Italy; ${ }^{8}$ Warwick Medical School, \\ University of Warwick, UK \\ Correspondence to Gaelle Hendrickx \\ (gaelle.hendrickx@kuleuven.be) \\ First received 13 Feb 2019, final revision \\ 29 Oct 2019, accepted 12 Nov 2019 \\ (c) The Authors 2020. This is an Open \\ Access article, distributed under the \\ terms of the Creative Commons \\ Attribution-NonCommercial- \\ NoDerivatives licence (http:// \\ creativecommons.org/licenses/by-nc- \\ nd/4.0/), which permits non- \\ commercial re-use, distribution, and \\ reproduction in any medium, provided \\ the original work is unaltered and is \\ properly cited. The written permission of \\ Cambridge University Press must be \\ obtained for commercial re-use or in \\ order to create a derivative work.
}

The transition from child and adolescent to adult mental health services for young people with mental health problems is of international concern. Despite the high prevalence of mental disorders during adolescence and their tendency to continue during adulthood, the majority of young people do not experience continuity of care. The aim of this review paper is to unravel the complexity of transitional mental healthcare to clinicians, policy makers and mental health service managers, and to address challenges to a smooth transition process at all levels.

\section{Declaration of interest None.}

Keywords Patients; psychosocial interventions; carers.
Young people's transition from child and adolescent mental health services (CAMHS) to adult mental health services (AMHS) has gained increasing international interest in recent years. At around the age of 16-18 years, young people with chronic mental health problems are confronted with two simultaneous transitions: a situational transition (from CAMHS to AMHS) and a developmental transition (to adulthood). ${ }^{1}$

Transition is defined as an efficient, planned, patientoriented process that meets the medical, psychosocial and educational/occupational needs of young people with chronic conditions. ${ }^{2}$ Additionally, the developmental perspective is important in conceptualising transition as young people, those aged 16-24 years, are confronted with changes in various life domains.,

The transition age refers to the age at which the care of the young person in CAMHS is continued in AMHS, and in most cases, concerns young people in the 16-18 year age range. The following findings linked with psychopathology 
are relevant for transitional care at this time point: first, psychopathology has a high persistence from an early age into adulthood $^{5}$; second, the onset of many psychopathologies has been shown to coincide with the transition age., Critical changes in the brain occur during late adolescence, which make this age group more vulnerable for developing psychopathology. ${ }^{7}$

Moreover, $75 \%$ of all psychiatric disorders in adults start before 24 years of age, and $50 \%$ before 14 years of age. ${ }^{8}$ However, there is a discord in the pattern of increased risk of psychopathology in young people and mental health service use. ${ }^{1,9}$ Older adolescents access care far less (28.9\%) than 13- to 16-year-olds (50.9\%) or adults over 26 years $(41.1 \%){ }^{10,11}$ Furthermore, the gap between CAMHS and AMHS, the so-called 'transition gap', results in a clear discontinuity of care. A sizeable number of youth and young adults who 'fall' in this transition gap access adult services at a later point in time, when more serious and chronic problems have developed. ${ }^{12,13}$

The care gap affects not only the young people, but also their families, communities and society as a whole. Mental health problems are associated with poorer physical health and poorer functioning in the social, educational and economic life domains. ${ }^{14}$ At the societal level, the presence of a mental disorder during childhood leads to a 10-fold higher health cost during adulthood compared with children without mental health problems. ${ }^{15}$ To date, it is unclear what the real societal impact of the care gap in late adolescence is and to what extent adequate transitional care reduces this impact.

The aim of this paper is to summarise the new insights and developments investigated since the review of Singh in 2009. ${ }^{12}$ Furthermore, this paper includes research on youth mental health services and shared management components, which is one of the limitations of the review of Paul et al. ${ }^{16}$

Continuation of care is a complex process, with important players at the policy and organisational level, service level and at the level of individuals: patients, their families and healthcare providers. However, care discontinuity cannot be explained by only one level. ${ }^{4,17}$ This paper attempts to unravel the complexity of transitional mental healthcare to clinicians, policy makers and mental health service managers, and to address challenges to a smooth transition process at all levels. The advantage of splitting the findings according to the three levels makes the extensive research regarding transition more manageable. Furthermore, it emphasises the complexity of the topic, but also makes clear that solutions are possible at every level.

\section{Challenges regarding transitional mental healthcare}

\section{Policy and organisational level}

Improving transitional care has been on the policy agenda in different European countries for some time now; however, it has been one of a number of competing priorities. Furthermore, there has been a gap between policy and implementation in practice, insufficient research regarding transition, and a lack of transition protocols guiding transitional care of service providers. ${ }^{16}$

Historically, the way in which mental health services have been structured, with separate facilities for children/adolescents and adults is a significant bottleneck to transitional care. $^{13}$ Eligibility thresholds for referral to CAMHS and AMHS often differ; thereby causing young people to fall through the care gap. ${ }^{4,18}$ Furthermore, the CAMHS-AMHS interface is characterised by different cultural approaches, a lack of communication and doubts about AMHS staff being proficient in managing young peoples' care. ${ }^{19}$

Waiting lists form another major barrier in the provision of mental healthcare to youth and young adults. ${ }^{20,21}$ As a result, young people presenting with a mental health problem at 17 years of age are often referred directly to AMHS because the waiting time for CAMHS coincides or exceeds their 18th birthday, i.e. the transition boundary. Adult services, in turn, hesitate to treat these young people before 18 years of age, because the expertise or the adjusted setting for this patient group is lacking, or because internal or external regulations prevent care providers from forming a treatment plan. Young people who have attended CAMHS but find themselves on a long waiting list for AMHS - usually with no interim support in place - describe this as highly anxiety-provoking. ${ }^{22}$

Another challenge is the lack of training requirements for care providers and variations in the content of training programmes. ${ }^{23,24}$ The knowledge clinicians in either child/ adolescent or adult speciality acquire about young people largely depends on whether they are provided with adequate training on psychopathology of adolescents. A lack of confidence amongst mental health practitioners to work with young people in transition is an area of concern frequently identified by young people. $^{22}$

Healthcare financing is another policy challenge affecting the care trajectories of young people. Gaining financial responsibility at the age of majority can have a real effect on the provision of care in some countries. Differences in financial benefits for minors versus adults may relate to the cost of a consultation, the possibility to organise family therapy or the reimbursement of medication. Some health insurance policies provide partial reimbursement for therapy sessions for young people up to 18 years of age, but not for (young) adults. Hence, although they have reached the age of majority and the ability to organise their mental healthcare independently, young people often still remain financially dependent on their parents to pay for their care. If there is a serious disruption in the parent-child relationship, the continuation of care at a new mental health service may be in jeopardy.

\section{Service level}

Different treatment approaches at CAMHS and AMHS pose a significant challenge. In CAMHS, treatment is reported to be more family-oriented and holistic, inherent to the legal position of the parents, whereas in adult psychiatry, individual patients and their symptoms are the main focus. These differences are described by young people and their families as an important reason for discontinuing care in AMHS. ${ }^{25}$ This perception may also lead to hesitance among CAMHS clinicians to refer young people. ${ }^{16}$ In addition, the lack of 
common registration and information systems hampers the exchange of information between services. ${ }^{17}$

\section{Level of the individual}

\section{Youth and young adults}

Characteristics specific to young people can influence the transition process. Having a severe and enduring mental illness, e.g. schizophrenia, enhances the chance of being referred to AMHS, whereas having a neurodevelopmental disorder decreases the chance of being referred. Moreover, receiving medication, having a history of hospital admissions and living with both parents or independently are all variables that can determine whether a young person is more likely to be referred. ${ }^{4,26-28}$

Even if a transition to adult services has been carefully planned, a young person's urge for autonomy and selfdetermination may influence their care trajectory. For example, the young person can decide to abandon psychological care or to not make the transition to AMHS, even when a referral has been made., ${ }^{4,26,28,29}$ The reasons for this are diverse: young people want to solve their problems themselves, or they may not want to repeat their story to a new clinician. The lack of information about mental healthcare, the stigma associated with mental health problems, ${ }^{30,31}$ anxiety about how confidentiality is handled and the physical accessibility of mental health services can all act as barriers to seeking help or accessing care. ${ }^{32}$ Young people have also suggested that further investments should be made to improve the accessibility of mental healthcare and have pointed out to the importance of e-health. ${ }^{17}$

Furthermore, service (dis)engagement is influenced by an identity change that accompanies the transition from CAMHS to AMHS. Besides adopting an adult identity, transitioning to AMHS implies adopting a new illness identity. ${ }^{31}$ Although CAMHS is associated with temporary psychopathology, AMHS is associated with having a severe and enduring mental illness, as this is often the prerequisite for being referred to or accepted by AMHS. ${ }^{4}$ Disengagement can be attributed to failure in adopting a new illness identity, an illness identity that is incompatible with AMHS service remit or fractious professional relationships between CAMHS and AMHS during the transition, which causes anxiety and uncertainty to the young person. ${ }^{31}$

Although young people want to make autonomous decisions and are concerned about the confidentiality of information, the loss of parental or other psychosocial support is an important negative factor for care continuation or adequate help-seeking behaviour. ${ }^{32}$

The relationship of trust with the CAMHS clinician must not be forgotten, as at the transition to adult services this relationship comes to an end. Entering into a new social and trust relationship at an AMHS can be daunting for young people. On the other hand, a positive relationship with the new clinician can enable the development of other positive relationships. ${ }^{33}$

\section{The parents and important others}

The parents' position changes the moment a young person becomes an adult, as their legal right to be involved in the care for their child is no longer there. Furthermore, because of the distinct service cultures, CAMHS and AMHS clinicians' training regarding family involvement differs considerably; in adult psychiatry, the focus is more on the individual, not the family. ${ }^{25,34}$

The need to give a young person autonomy to make their own decisions regarding treatment can be a difficult process for the parents, who may also require additional support. ${ }^{35}$ Many parents and carers would like to remain involved in the treatment, although they respect their child's wishes and their right to privacy. ${ }^{36,37}$ They also want psychoeducation about how to deal with their child and to attend parent support groups where they can benefit from increased knowledge, shared recognition and exchange of experiences. $^{38}$

\section{The clinician}

Mental healthcare transition should be a planned and efficient process. This implies starting on time to prepare individuals and their families for the transition. Some authors state that this process should start at 14 years of age, ${ }^{39}$ whereas others stress that it should start at least 1 year before the transition boundary. ${ }^{40}$ For the process to be efficient, by the time the young person reaches the transition boundary it should be clear whether they need further care or not, and whether this care will be continued in CAMHS or whether a referral to AMHS, or another type of service (e.g. private practice), is appropriate. In any case, the clinician should consider all these options to make the best possible decision.

To date, there is no consensus about this decision-making process, and the follow-up trajectory of the young person thus depends on the practitioner's clinical judgement. Because of the lack of transition protocols, this clinical judgement is not usually based on a structured assessment of transitionrelevant factors, such as severity of symptoms, the patient's motivation regarding further mental healthcare and the risk and protective factors in several psychosocial domains. ${ }^{13}$ CAMHS and AMHS should, therefore, be supported in the initiation, advancement and supervision of the transition process. The National Institute for Health and Care Excellence and Cleverley et al have produced guidelines on transition. ${ }^{41,42}$ The Managing the Link and Strengthening Transition from Child to Adult Mental Healthcare (MILESTONE) project developed an instrument for assessing transition, the Transition Readiness and Appropriateness Measure, a process called managed transition, which uses the Transition Readiness and Appropriateness Measure to guide clinicians' actions, and training regarding transition. ${ }^{43,44}$

The transition process is also influenced by professional relationships between CAMHS and AMHS. Clinicians' decisions regarding referrals may depend on the (not always comprehensive) knowledge they have of the other care provider and their prior experience with the service and clinician. $^{45}$ Furthermore, incompatible beliefs about who is responsible for the different steps during the transition process, lack of confidence in AMHS staff in managing young people and different cultural approaches in service delivery may also impede the transition process. ${ }^{18,46}$ 


\section{Improving transition through specific interventions}

Some of the above-mentioned challenges provide directions as to what should be done in clinical practice and at policy level to improve the transition process. On the other hand, effect studies are lacking and there is a need for longitudinal research about different transition trajectories and health outcomes. ${ }^{16,47}$ Although care trajectories, transition experiences and quality of transition have been investigated within the UK, ${ }^{1}$ Ireland, ${ }^{28,46}$ the USA and Australia, ${ }^{16}$ no research has been performed about the care and transition trajectories (both the experiences and the quality) in relation to their effects on mental health in the long term. The MILESTONE project contains a prospective study on the longitudinal outcomes and experiences of young people reaching the transition boundary within eight different European countries, taking into account differences in the organisation of mental health systems, the age at which transition takes place and the available services. ${ }^{43,44}$ The MILESTONE study will result in evidence- and practice-based guidelines that clinicians can follow to support their decision-making and direct their actions.

To prevent young people from falling through the care gap and to tailor services to their specific needs, new service models have been developed. Examples include mental health services in Australia, Canada and some European countries that target the age group of 0-25 years. Besides solely focusing on mental health, these services take into account all aspects of psychosocial functioning. ${ }^{4-50}$ Despite the aim of trying to solve the problem of a shortage of tailored services for this target group, some of these services are faced with an additional transition boundary: the first around 12 years of age and the second around 25 years of age, both of which need to be optimally managed. At the current time, it is too early to conclude if these models provide an answer to the longstanding problems of transition barriers.

An alternative approach to bridge the transition gap is by improving the liaison between CAMHS and AMHS, but keeping services as they currently exist. To achieve this, diverse models to enhance joint-working between services, including transition clinics and transition coordinators have been suggested. ${ }^{51-54}$

\section{Improving clinical practice}

Policy makers should consider implementing the topic of transition in the training program of clinicians as $94 \%$ of European psychiatric trainees indicated further training regarding transition is necessary. ${ }^{23}$ Furthermore the distinct split between CAMHS and AMHS should be revised as well as the separate funding, which may hamper collaborative efforts. $^{13,42}$

To ensure that the transition process is better managed, the transition should be mentioned to the young person well in advance, ${ }^{17,41,42}$ whereby the young person should be involved in the decision-making during all phases of the process. $^{22,42,55}$ Guidelines and criteria regarding optimal transition can guide clinicians during their clinical practice., ${ }^{4,41,42}$
Furthermore, standardised assessment of the young persons' needs when approaching the transition boundary should become routine, although it is rarely done nowadays. ${ }^{13}$

\section{Conclusion}

The transition from CAMHS to AMHS is an important process for young people with mental health problems. Literature shows that continuation of care is a complex process, with important players at policy and organisational levels, service level and at the level of individuals: patients, their families and healthcare providers. At the moment, specific programmes for young people are being developed. However, research such as the MILESTONE project is needed to support these interventions in an evidence-based manner.

\section{Funding}

The MILESTONE project has received funding from the European Union's Seventh Framework Programme for research, technological development and demonstration under grant agreement no. 602442. This paper reflects only the authors' views and the European Union is not liable for any use that may be made of the information contained therein. The funding body has had no role in the study design, in the writing of the protocol or in the decision to submit the paper for publication.

S.P.S. is part-funded by the National Institute for Health Research (NIHR) Collaboration for Leadership in Applied Health Research and Care WM (CLAHRC-WM). The views expressed are those of the authors and not necessarily those of the CLAHRC-WM collaborative organisations, the NIHR or the Department of Health.

\section{Acknowledgements}

We would like to thank Marc Hermans, past president of the European Union of Medical Specialists (UEMS) board of psychiatry, for inviting us to join the UEMS working group regarding transition. We are also very grateful for the whole UEMS working group on transition for their interest and plans to make transition part of the training of psychiatrists throughout Europe. Furthermore, we would like to thank all members of the MILESTONE consortium.

\section{About the authors}

Gaelle Hendrickx is a PhD student at Department of Neurosciences, Centre for Clinical Psychiatry, KU Leuven, Belgium, and a research associate with the MILESTONE consortium. Veronique De Roeck is a is a researcher at the Department of Neurosciences, KU Leuven, Belgium, and a research associate with the MILESTONE consortium. Athanasios Maras is Director of Yulius Academy, Yulius Mental Health Organization, The Netherlands, and a principal investigator with the MILESTONE consortium. Gwen Dieleman is a child and adolescent psychiatrist and clinical research coordinator at Department of Child and Adolescent Psychiatry and Psychology, Erasmus Medical Center, The Netherlands, and a principal investigator with the MILESTONE consortium. Suzanne Gerritsen is a PhD student at the Department of Child and Adolescent Psychiatry and Psychology, Erasmus Medical Center, The Netherlands, and a research associate with the MILESTONE consortium. Diane Purper-Ouakil is a psychiatrist at the Child and Adolescent Psychiatry Unit of the University Hospital of Montpellier, France, and a principal investigator with the MILESTONE consortium. Frederick Russet is a psychologist at the Child and Adolescent Psychiatry Unit of the University Hospital of Montpellier, France, and research associate with the MILESTONE consortium. Renate Schepker is a psychiatrist at the Centre for Psychiatry South-Wuerttemberg, Germany, and a principal investigator with the MILESTONE consortium. Giulia Signorini is a researcher at the Psychiatric Epidemiology and Evaluation Unit of Saint John of God Clinical Research Center, Italy, and research associate with the MILESTONE consortium. Swaran Preet Singh is Head of 
Mental Health and Wellbeing at Warwick Medical School, University of Warwick, UK, and Chief Investigator with the MILESTONE consortium. Cathy Street is the Patient and Public Involvement Lead at Warwick Medical School, University of Warwick, UK, and at the MILESTONE consortium. Helena Tuomainen is a senior research fellow at Warwick Medical School, University of Warwick, UK, and Scientific Research Manager with the MILESTONE consortium. Sabine Tremmery is a professor at the Department of Neurosciences, KU Leuven, Belgium, and a principal investigator with the MILESTONE consortium.

\section{References}

1 Singh SP, Paul M, Ford T, Kramer T, Weaver T. Transitions of care from child and adolescent mental health services to adult mental health services (TRACK study): a study of protocols in Greater London. BMC Health Serv Res 2008; 8: 135.

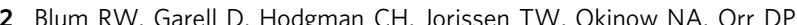
et al. Transition from child-centered to adult health-care systems for adolescents with chronic conditions: a position paper of the Society for Adolescent Medicine. J Adolesc Health 1993; 14(7): 570-6.

3 Arnett JJ. Emerging adulthood. A theory of development from the late teens through the twenties. Am Psychol 2000; 55(5): 469-80.

4 Singh SP, Paul M, Ford T, Kramer T, Weaver T, McLaren S, et al. Process, outcome and experience of transition from child to adult mental healthcare: multiperspective study. Br J Psychiatry 2010; 197(4): 305-12.

5 Reef J, Diamantopoulou S, Meurs I, Verhulst F, Ende J. Predicting adult emotional and behavioral problems from externalizing problem trajectories in a 24-year longitudinal study. Eur Child Adolesc Psychiatry 2010; 19(7): 577-85.

6 Kessler CR, Amminger PG, Aguilar-Gaxiola BS, Alonso BJ, Lee BS, Üstün BT. Age of onset of mental disorders: a review of recent literature. Curr Opin Psychiatry 2007; 20(4): 359-64.

7 de Girolamo G, McGorry PD, Sartorius N. Age of Onset of Mental Disorders: Etiopathogenetic and Treatment Implications. Springer, 2018.

8 Kessler RC, Chiu WT, Demler O, Walters EE. Prevalence, severity, and comorbidity of 12-month DSM-IV disorders in the National Comorbidity Survey Replication. Arch Gen Psychiatry 2005; 62(6): 617.

9 Vanclooster C, Vanderhaegen J, Bruffaerts R, Hermans K, Van Audenhove $\mathrm{C}$. Inschatting van de behoefte aan geestelijke gezondheidszorg. Steunpunt Welzijn, Volksgezondheid en Gezin, 2013.

10 Wang PS, Lane $M$, Olfson $M$, Pincus $H$, Wells $K$, Kessler RC Twelve-month use of Mental Health Services in the United States results from the National Comorbidity Survey Replication. Arch Gen Psychiatry 2005; 62(6): 629-40.

11 Copeland WE, Shanahan L, Davis M, Burns BJ, Angold A, Costello EJ. Increase in untreated cases of psychiatric disorders during the transition to adulthood. Psychiatr Serv 2015; 66(4): 397-403.

12 Singh SP. Transition of care from child to adult mental health services: the great divide. Curr Opin Psychiatry 2009; 22(4): 386-90.

13 Signorini G, Singh SP, Marsanic VB, Dieleman G, Dodig-Curkovic K, Franic $T$, et al. The interface between child/adolescent and adult mental health services: results from a European 28-country survey. Eur Child Adolesc Psychiatry 2018; 27(4): 501-11.

14 Singleton N, Lewis G. Better or Worse: A Longitudinal Study of the Mental Health of Adults Living in Private Households in Great Britain: Report Based on Surveys Carried out by the Office for National Statistics in 2000 and 2001 for the Department of Health and the Scottish Executive Health Department. The Stationery Office, 2003.

15 Suhrcke M, Pillas D, Selai C. Economic Aspects of Mental Health in Children and Adolescents. World Health Organization Regional Office for Europe, 2008.

16 Paul M, Street C, Wheeler N, Singh SP. Transition to adult services for young people with mental health needs: a systematic review. Clin Child Psychol Psychiatry 2015; 20(3): 436-57.

17 Coppens E, Vermet I, Knaeps J, De Clerck M, De Schrijver I, Matot JP, et al. ADOCARE - A Preparatory Action Related to the Creation of an EU
Network of Experts in the Field of Adapted Care for Adolescents with Mental Health Problems. European Commission, 2015.

18 Lamb C, Murphy M. The divide between child and adult mental health services: points for debate. Br J Psychiatry 2013; 202(54): s41-4.

19 McLaren S, Belling R, Paul M, Ford T, Kramer T, Weaver T, et al. Talking a different language': an exploration of the influence of organizational cultures and working practices on transition from child to adult mental health services. BMC Health Serv Res 2013; 13: 254.

20 Paul M, Ford T, Kramer T, Islam Z, Harley K, Singh SP. Transfers and transitions between child and adult mental health services. $\mathrm{Br} J$ Psychiatry 2013; 202(s54): s36-40.

21 Vandenbroeck P, Dechenne R, Becher K, Eyssen M, Van Den Heede K. Recommendations for the organization of mental health services for children and adolescents in Belgium: use of the soft systems methodology. Health Policy 2014; 114(2-3): 263-8.

22 Street C, Walker L, Tuffrey A, Wilson A. Transition between differen UK mental health services: young people's experiences on what makes a difference. J Clin Psychiatry Cognitive Psychol 2018; 2: 1-5.

23 Hendrickx G, De Roeck V, Russet F, Dieleman G, Franic T, Maras A et al. Transition as a topic in psychiatry training throughout Europe: trainees' perspectives. Eur Child Adolesc Psychiatry 2019; 9: 1-9.

24 Russet F, Humbertclaude V, Dieleman G, Dodig-Curkovic K, Hendrickx G, Kovac V, et al. Training of adult psychiatrists and child and adolescent psychiatrists in Europe: a systematic review of training characteristics and transition from child/adolescent to adult mental health services. BMC Med Educ 2019; 19: 204.

25 Reale L, Bonati M. Mental disorders and transition to adult mental health services: a scoping review. Eur Psychiatry 2015; 30(8): 932-42.

26 McNicholas F, Adamson M, McNamara N, Gavin B, Paul M, Ford T, et al. Who is in the transition gap? Transition from CAMHS to AMHS in the Republic of Ireland. Ir J Psychol Med 2015; 32(1): 61-9.

27 Perera RH, Rogers SL, Edwards S, Hudman P, Malone C. Determinants of transition from child and adolescent to adult mental health services: a Western Australian pilot study. Aust Psychol 2017; 52(3): 184-90.

28 Leavey G, McGrellis S, Forbes T, Thampi A, Davidson G, Rosato M, et al. Improving mental health pathways and care for adolescents in transition to adult services (IMPACT): a retrospective case note review of social and clinical determinants of transition. Soc Psychiatry Psychiatr Epidemiol 2019; 54: 955-63.

29 Breland DJ, McCarty CA, Zhou C, McCauley E, Rockhill C, Katon W et al. Determinants of mental health service use among depressed adolescents. Gen Hosp Psychiatry 2014; 36(3): 296-301.

30 Davis M, Butler M. Service System Supports during the Transition from Adolescence to Adulthood: Parent Perspectives. Implementation Science and Practice Advances Research Center Publications, 2002 (https:// escholarship.umassmed.edu/psych_cmhsr/435/).

31 McNamara N, Coyne I, Ford T, Paul M, Singh SP, McNicholas F. Exploring social identity change during mental healthcare transition. Eur J Soc Psychol 2017; 47: 889-903.

32 Gulliver A, Griffiths KM, Christensen H. Perceived barriers and facilitators to mental health help-seeking in young people: a systematic review. BMC Psychiatry 2010; 10: 113.

33 Hiles D, Moss D, Wright J, Dallos R. Young people's experience of social support during the process of leaving care: a review of the literature. Child Youth Serv Rev 2013; 35(12): 2059-71.

34 Singh SP, Evans N, Sireling L, Stuart H. Mind the gap: the interface between child and adult mental health services. Psychiatr Bull 2005; 29: 292-4.

35 Rodriguez-Meirinhos A, Antolin-Suarez L, Oliva A. Support needs of families of adolescents with mental illness: a systematic mixed studies review. Arch Psychiatr Nurs 2018; 32(1): 152-63.

36 Jivanjee P, Kruzich JM, Gordon LJ. The age of uncertainty: parent perspectives on the transitions of young people with mental health difficulties to adulthood. J Child Fam Stud 2009; 18(4): 435-46.

37 Coyne I, McNamara N, Healy M, Gower C, Sarkar M, McNicholas F. Adolescents' and parents' views of Child and Adolescent Mental 
Health Services (CAMHS) in Ireland. J Psychiatr Ment Health Nurs 2015; 22(8): $561-9$.

38 Stapley E, Midgley N, Target M. The experience of being the parent of an adolescent with a diagnosis of depression. J Child Fam Stud 2016; 25(2): 618-30.

39 McDonagh JE. Transition of care: how should we do it? Paediatr Child Health 2007; 17(12): 480-4.

40 Suris JC, Rutishauser C, Akre C. Does talking about it make a difference? Opinions of chronically ill young adults after being transferred to adult care. Arch Pediatr 2015; 22(3): 267.

41 National Institute for Health and Care Excellence (NICE). Transition from Children's to Adults' Services for Young People Using Health or Social Care Services, Guideline 43. NICE, 2016 (https://www.nice.org.uk/guidance/ ng43)

42 Cleverley K, Rowland E, Bennett K, Jeffs L, Gore D. Identifying core components and indicators of successful transitions from child to adult mental health services: a scoping review. Eur Child Adolesc Psychiatry 2018, in press. doi:10.1007/s00787-018-1213-1

43 Singh SP, Tuomainen H, de Girolamo G, Maras A, Santosh P, McNicholas F, et al. Protocol for a cohort study of adolescent mental health service users with a nested cluster randomised controlled trial to assess the clinical and cost-effectiveness of managed transition in improving transitions from child to adult mental health services (the MILESTONE study). BMJ Open 2017; 7(10): e016055.

44 Tuomainen $\mathrm{H}$, Schulze $U$, Warwick J, Paul M, Dieleman G, Franic T, et al. Managing the link and strengthening transition from child to adult mental health care in Europe (MILESTONE): background, rationale and methodology. BMC Psychiatry 2018; 18: 167.

45 Aebi M, Kuhn C, Metzke C, Stringaris A, Goodman R, Steinhausen H-C. The use of the development and well-being assessment (DAWBA) in clinical practice: a randomized trial. Eur Child Adolesc Psychiatry 2012; 21 (10): 559-67.

46 McNamara N, McNicholas F, Ford T, Paul M, Gavin B, Coyne I, et al. Transition from child and adolescent to adult mental health services in the Republic of Ireland: an investigation of process and operational practice. Early Interv Psychiatry 2014; 8(3): 291-7.
47 Embrett $M$, Randall G, Longo $C$, Nguyen T, Mulvale G. Effectiveness of health system services and programs for youth to adult transitions in mental health care: a systematic review of academic literature. Adm Policy Ment Health 2016; 43(2): 259-69.

48 McGorry PD, Goldstone SD, Parker AG, Rickwood DJ, Hickie IB. Cultures for mental health care of young people: an Australian blueprint for reform. Lancet Psychiatry 2014; 1(7): 559-68.

49 Rickwood DJ, Mazzer KR, Telford NR, Parker AG, Tanti CJ, McGorry PD. Changes in psychological distress and psychosocial functioning in young people visiting headspace centres for mental health problems. Med J Aust 2015; 202(10): 537-42.

50 Halsall T, Manion I, Iyer SN, Mathias S, Purcell R, Henderson J. Trends in mental health system transformation: Integrating youth services within the Canadian context. Healthc Manage Forum 2019; 32(2): 51-5.

51 Maitra B, Jolley A. Liaison between child and adult psychiatric services. In Family Matters: Interface Between Child and Adult Mental Health (eds P Reder, M McClure, A Jolley): 285-302. Routledge, 2000.

52 Cappeli M, Davidson S, Racek J, Leon S, Vloet M, Tataryn K, et al. Transitioning youth into adult mental health and addiction services: an outcomes evaluation of the Youth Transition Project. I Behav Health Serv Res 2016; 43(4): 597-610.

53 Henderson JL, Cheung A, Cleverley K, Chaim G, Moretti ME, de Oliveira $\mathrm{C}$, et al. Integrated collaborative care teams to enhance service delivery to youth with mental health and substance use challenges: protocol for a pragmatic randomised controlled trial. BMJ Open 2017; 7(2): e014080.

54 Health and Social Care Advisory Service (HASCAS). CAMHS to Adult Transition: A Literature Review for Informed Practice. Health and Social Care Advisory Service (HASCAS) Tools for Transition. Department of Health and HASCAS, 2006

55 Broad KL, Sandhu VK, Sunderji N, Charach A. Youth experiences of transition from child mental health services to adult mental health services: a qualitative thematic synthesis. BMC Psychiatry 2017; 17: 380.

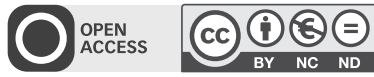

